\title{
Clinical characteristics and outcomes of the oldest old surgical critical
}

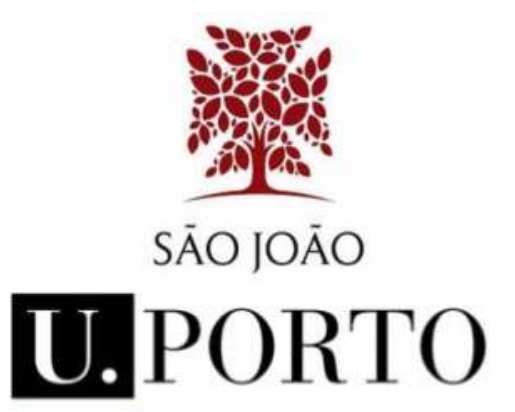

FMUP FACULDADE DE MEDICINA UNIVERSIDADE DO PORTO

\section{patient}

Luís Guilherme Casimiro¹, Luciana Ferreira², Mónica Oliveira ${ }^{1}$, Diana Silva ${ }^{1}$, Alice Santos $^{1,2}$, Fernando Abelha $^{1,2}$

1 - Centro Hospitalar São João E.P.E., Dept of Anaesthesia, Porto, Portugal

2 - Faculty of Medicine, University of Porto, Dept of Anaesthesia, Porto, Portugal

Eurroanaesthesia

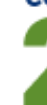

2018

\section{Introduction}

The elderly population might be subclassified in three categories: young elderly 65-75 years, older elderly 75-85 years, very elderly $>85$ years $^{(1)}$. The percentage of very elderly patients has been rising in the last years, as well as the number of surgeries performed in this population. Therefore, there's also been an increase of the mean age of patients admitted in the Surgical Intensive Care Unit (SICU).(1,2)

\section{Study purpose}

To compare surgical outcomes evaluating the impact of age and functional status on SICU admitted patients.

\section{Methods}

Study-type: observational retrospective study

Population: Patients admitted at SICU

Temporal interval: January 2006 to July 2013

Inclusion criteria:Patients submitted to non-cardiac, nonneurologic elective and emergent surgery admitted at SICU

\section{Exclusion criteria:}

- Age <65;

- length of stay < $12 \mathrm{~h}$ in SICU;

- patients readmitted in the context of initial admission in the study period

Patients aged $>85$ years were considered to be "very elderly" (VEG - very elderly group)

Patient's demographics, intraoperative and postoperative data were collected. Descriptive analysis was performed and the Mann-Whitney $U$ test, Fisher's exact test or Chi-square were used.

\section{Results}

2331 patients included

\begin{tabular}{|c|c|}
\hline EG & VEG \\
\hline 2205 & 126 \\
\hline $94,6 \%$ & $5,4 \%$ \\
\hline
\end{tabular}

Table 1 - Univariate analysis of patients demographics and clinical values

\begin{tabular}{|c|c|c|c|}
\hline Variables & EG & VEG & $\mathrm{p}$ value \\
\hline Male gender, $n(\%)$ & $1400(63.5)$ & $52(41.3)$ & $<0.001$ \\
\hline Admission- Elective surgery, $\mathrm{n}(\%)$ & $1960(88.8)$ & $98(77.7)$ & $<0.001$ \\
\hline History of isquemic heart disease, $\mathrm{n}(\%)$ & $421(19.1)$ & $21(16.7)$ & 0.499 \\
\hline History of congestive heart disease, $n(\%)$ & $462(21)$ & $45(35.7)$ & $<0.001$ \\
\hline History of cerebrovascular disease, $n(\%)$ & $412(18.7)$ & $20(15.9)$ & 0.430 \\
\hline Preoperative insuline therapy $n(\%)$ & $139(6.3)$ & $7(5.6)$ & 0.736 \\
\hline Chronic renal failure, $\mathrm{n}(\%)$ & $169(7.7)$ & $14(11.1)$ & 0.162 \\
\hline $\mathrm{RCRI}>2, \mathrm{n}(\%)$ & $241(10.9)$ & $13(10.3)$ & 0.083 \\
\hline Organ insufficiency, n (\%) & $389(17.6)$ & $31(24.6)$ & 0.048 \\
\hline Body temperatura, median (P25-75) & $35.7(34.5-36.0)$ & $35,6(34.6-36.0)$ & 0.812 \\
\hline Heart rate, median (P25-75) & $85.0(70.0-96.0)$ & $78.5(65.0-93.0)$ & 0,589 \\
\hline Respiratory rate, mediana (P25-75) & $14.0(12.0-16.0)$ & $14,0(12.0-16.0)$ & 0.621 \\
\hline Systolic blood pressure, median (P25-75) & $\begin{array}{c}122.0(102.0- \\
145.0) \\
\end{array}$ & $121.5(99.8-140.5)$ & 0.082 \\
\hline Mean blood pressure, median (P25-75) & $85.0(70.0-96.0)$ & $78,5(65.0-93.0)$ & 0.015 \\
\hline Hematocrit,median (P25-75) & $33.0(29.5-36.0)$ & $32.3(29.9-35.6)$ & 0.281 \\
\hline Urea, median (P25-75) & $32.0(22.0-46.0)$ & $36.0(24.8-50.3)$ & 0.046 \\
\hline Serum creatinine, median (P25-75) & $9.0(7.0-12.0)$ & $9.95(7.0-13.0)$ & 0.288 \\
\hline Total bilirrubin, median (P25-75) & $5.0(1.0-7.0)$ & $4.0(1.0-7.0)$ & 0.118 \\
\hline Leucocytes, median (P25-75) & $10.4(7.6-13.4)$ & $10.6(7.7-13.0)$ & 0.894 \\
\hline Serum sodium, median (P25-75) & $\begin{array}{c}140.0(130.0- \\
142.0) \\
\end{array}$ & $139.0(137.0-142.0)$ & 0.293 \\
\hline Serum potassium, median (P25-75) & $3.8(3.4-4.1)$ & $3.8(3.3-4.0)$ & 0.330 \\
\hline Ventilated, n (\%) & $651.0(29.5)$ & $32.0(25.4)$ & 0.322 \\
\hline $\mathrm{FiO} 2$, median (P25-75) & $0.4(0.3-0.4)$ & $0.4(0.3-0.4)$ & 0.148 \\
\hline $\mathrm{pH}$, median (P25-75) & $7.4(7.3-7.4)$ & $7.4(7.3-7.4)$ & 0.648 \\
\hline $\mathrm{PaO} 2$, median (P25-75) & $\begin{array}{c}100.0(100.0- \\
109.0)\end{array}$ & $100.0(100.0-105.0)$ & 0.695 \\
\hline PaCO2, median (P25-75) & $40.0(35.0-45.0)$ & $41.0(35.7-45.0)$ & 0.096 \\
\hline Serum bicarbonate, median (P25-75) & $22.0(21.0-24.0)$ & $22.0(21.0-24.0)$ & 0.080 \\
\hline \multicolumn{4}{|c|}{ Table 2 - Severity of disease scores, length of stay and mortality } \\
\hline Variables & EG & VEG & $\mathrm{p}$ value \\
\hline SAPS II, median (P25-75) & 22.2(17.8-28.9) & $26.7(20-33.9)$ & $<0.001$ \\
\hline APACHE II, median (P25-75) & $10.0(8.0-13.0)$ & $12.0(9.0-15.3)$ & $<0.001$ \\
\hline Time in SICU (hours), median (P25-75) & $20(16-41)$ & $20(16-43)$ & 0.898 \\
\hline MCE, $n(\%)$ & $74.0(3.4)$ & $1.0(0.8)$ & 0.079 \\
\hline SICU mortality, n (\%) & $37.0(2.0)$ & $6.0(5.0)$ & 0.026 \\
\hline Hospital mortality, $\mathrm{n}(\%)$ & $204.0(9.0)$ & $21.0(17.0)$ & 0.006 \\
\hline
\end{tabular}

EG- Eldery Group; VEG- Very Eldery Group; RCRI -Revised Cardiac Risk Index; APACHE II-Acute Physiology and Chronic Health Evaluation; SAPS II- Simplified Acute Physiology Score; MCE -Major cardiac event; SICU - surgical intensive care unit

\section{Discussion}

The very elderly population represents an important group of patients admitted in the operating room and consequently in the SICU. It's important to understand how they may differ from the other patients, to prevent further complications.

\section{Conclusions}

In our study, it was identified a higher mortality rate at SICU and hospital in this very elderly group, and a higher prevalence of organ failure. This increased mortality was confirmed by higher severity of disease scores SAPS II and APACHE II. 